Title of paper:

\title{
Morphological integration of mandible and cranium: Orthodontic implications
}

Running Title: Mandible and cranium integration

José Antonio Alarcón ${ }^{\mathrm{a},{ }^{*}}$, Markus Bastir ${ }^{\mathrm{b}}$, Ignacio García-Espona ${ }^{\mathrm{a}}$, Mario MenéndezNúñez ${ }^{\mathrm{a}}$, Antonio Rosas ${ }^{\mathrm{b}}$

${ }^{\text {a }}$ Department of Stomatology, Section of Orthodontics, Faculty of Odontology, University of Granada, Granada, Spain

b Paleoanthropology Group, Department of Paleobiology, Museo Nacional de Ciencias Naturales, CSIC, Madrid, Spain

*José Antonio Alarcón, Department of Stomatology, Faculty of Odontology, University of Granada, Campus Universitario de Cartuja, s/n 18071, Granada, Spain

Tel. and fax: +34 958201480

E-mail address: jalarcon@ugr.es

Keywords: covariation, geometric morphometrics, generalized linear model, skeletal Class II, III- malocclusion, facial pattern. 


\section{ABSTRACT}

Objectives: This study aimed at clarifying the morphological interactions among the cranial base, face, and mandible, to improve the assessment and treatment of skeletal malocclusions involving the mandible.

Design: Untreated adult subjects $(\mathrm{N}=187)$ were grouped according to standard cephalometric criteria of vertical and sagittal relationships. Geometric morphometrics were used to test the null hypothesis that integration patterns between the mandible and its associated basicranial and upper midfacial counterparts would be similar among various vertical and sagittal facial patterns.

Results: The null hypothesis was rejected for vertical groups, because the dolicho- and brachyfacial subjects showed significantly different integration patterns, but was accepted for sagittal groups, which showed identical covariation patterns. The morphological integration between the cranium-face and mandible were similarly high in the three skeletal classes, which explained the similarly large covariance between the two structures $(57.80 \%$ in Class II to 60\% in Class III).

Conclusions: Dolicho- and brachi-facial subjects showed specific and different craniumface and associated mandible configurations. The cranium-face configuration may have an important influence $(\sim 60 \%)$ on the generation of sagittal (anteroposterior) skeletal malocclusions. The remaining morphological component of the skeletal malocclusion ( 40\%) would be independent of this particular integration (PLS1) between the craniumface and mandible.

Keywords: covariation, geometric morphometrics, generalized linear model, skeletal Class II, III- malocclusion, facial pattern. 


\section{Introduction}

The term morphological integration (MI) ${ }^{1-3}$ implies that an evolutionary change in the morphology of one anatomical element is reflected by morphological changes in other elements. ${ }^{4}$ MI comprises a set of mechanisms that connect (integrate) elements of an anatomical system, quantify the associations between them, and provide measures of covariation to infer developmental or functional relationships. ${ }^{5}$ Many orthodontic treatments seek to affect the growth of the mandible. ${ }^{6-10}$ However, differences in cranium and mandible MI patterns between patients, depending on their sex, jaw skeletal relationship, or facial pattern, could result in divergent responses to orthopedic treatment. Therefore, accurate knowledge about the interdependence among craniofacial structures (e.g., mandible, face, and cranial base) is critical for therapeutic planning.

It is still unclear whether orthopedic treatments can alter the mandibular growth to a clinically significant degree. For example, the effect of functional appliances over condylar growth is a topic of long-standing controversy. ${ }^{7}$ The MI between the mandible and craniofacial system could be partially responsible for the basic skeletal setting that leads to a given sagittal or vertical malocclusion. It could also explain the relatively limited response of the mandible to orthopedic appliances.

Several quantitative studies have investigated MI in the human face. ${ }^{11-14}$ Some studies found significant features of integration between the cranium and mandible or some of its elements. ${ }^{15}$ However, the idea that the mandible is relatively independent of the cranium remains pervasive. In a study of adolescents without major malocclusion, McKane and Kean ${ }^{14}$ found minor or no covariation among the shapes of parts of the facial skeleton. Recent research about the MI of the modern human mandible during ontogeny concluded that the mandible has maintained a passive role in hominin skull evolution, playing "follow 
the leader" with the cranium. ${ }^{16}$ Enlow offered a compromise between these extreme viewpoints, proposing that a brain-to-basicranium-to-face (mandible) cascade of morphological influence leads to integration. ${ }^{17,18}$ Later studies partially confirmed some of these spatiotemporal interconnections. ${ }^{19,20}$ More recently, Wellens et al., ${ }^{21}$ found that the mandible and maxilla constitute one module, independent of the skull base.

The fact that a high integration degree between the mandible and the cranium could exist in some cases, but not in others, raises some questions: For example, what are the morphological pattern (i.e., shape-coordinated variation) and the quantitative pattern (i.e., the degree of covariation) of the mandible-cranium integration, and do these integration patterns differ among various craniofacial configurations (e.g., occlusal and facial patterns)?

The aim of this study was to quantify patterns of morphological covariation between the mandible and cranium in adult subjects with skeletal Class I, II, and III malocclusions, on the one hand, and meso- dolicho-, and brachyfacial configurations, on the other hand. The overall goal was to improve the assessment and treatment of skeletal malocclusions involving the mandible. Because conventional distance-angle cephalometric approaches present limitations for shape assessment, ${ }^{22,23}$ this study employed geometric morphometrics, which have been shown to be useful for investigating MI. ${ }^{24-26}$ The null hypothesis was that there would be no difference in the craniofacial-mandibular integration pattern between groups.

\section{Material and methods}

\subsection{Data sample}


This study included 187 Caucasian adult subjects (92 males; 95 females, age range, 20-30 years; mean, $25.6 \pm 4.2$ years) from Granada (southern Spain) who were randomly selected from a private dental office. Exclusion criteria included: craniofacial disorders such as cleft anomalies, craniosynostoses, or other syndromal diseases or congenital malformation, congenitally missing, supernumerary, or extracted teeth; and previous or current orthopedic or orthodontic treatment.

For all subjects, standard lateral cephalometric radiographs with the teeth in centric occlusion and with the head oriented horizontally with the Frankfort plane were taken with a cephalostat in accordance with standard cephalometric prodecures. The same digital x-ray device (Planmeca PM-2002 EC Proline Dental Pan X-Ray Machine, Helsinki, Finland), technician, focus-median $(150 \mathrm{~cm})$, and film-median $(10 \mathrm{~cm})$ plane distances were used for all radiographs. A reference ruler was shown on the cephalostat for exact measurement of the magnification factor.

Cephalograms were imported into tpsDIG 2.12 software (tpsSeries, J.F. Rohlf, SUNY Stony Brook; http://life.bio.sunysb.edu/morph/) to digitize 38 landmarks (2D) representing the morphology of the cranial floor, the midline cranial base, and the face, and 31 semilandmarks representing the morphology of the lower surface of the mandibular body and the contour of the bony chin-symphysis (Table and Fig. 1). All of these localizations were performed by the same examiner (J.A.A.). Paired bilateral landmarks were digitized by averaging the left and right sides.

Measurement errors were evaluated by multivariate analysis of variance (MANOVA) by repeated data recordings of 10 randomly selected subjects on 4 different days. No significant differences were found between the repeated samples (Wilks lambda 5 
0.00; F 5 1.69; df1, 25 138, 6, 47; P 5 0.2), indicating that the measurement errors were smaller than the sample variations.

\subsection{Geometric morphometrics and statistical analyses}

The degree of covariation patterns were quantified with a two-block partial least squares (PLS) analysis, ${ }^{27}$ by assessing correlations between the first PLS vector scores ${ }^{25}$ and the RV coefficient. ${ }^{28}$ The integration pattern was quantified by using Procrustes registered configurations along the PLS vectors of the corresponding blocks. ${ }^{29}$ Blocks 1 was the cranium (cranial base and face), and block 2 was the mandible.

Integration vectors for the full sample after correction for sexual dimorphism were calculated. Sex correction was performed by multivariate regression of shape on sex (dummy) and avoided assessment of integration patterns driven by male and female mean shape differences. Then, mesofacial (FMA between $20^{\circ}$ and $28^{\circ}, \mathrm{n}=97$ ), dolichofacial (FMA $>28^{\circ} ; n=49$ ), and brachyfacial (FMA $<20^{\circ} ; n=41$ ) patterns, and skeletal Class I (ANB angle between $0^{\circ}$ and $3^{\circ}, \mathrm{n}=88$ ), Class II (ANB angle $>3^{\circ} ; n=54$ ), and Class III (ANB angle $<0^{\circ} ; n=45$ ) malocclusions were distinguished, following standard orthodontic criteria (ANB angle and FMA angle -mandibular plane to the Frankfurt horizontal angle). ${ }^{30,31}$

To assess the overall similarity of integration patterns in different groups of facial patterns and skeletal classes, craniofacial and mandibular PLS1 scores were analyzed by a Generalized Linear Model (GLM). ${ }^{32}$ We considered the overall correlation between the craniofacial and the mandibular PLS scores as principal factor as well as a group factor with three levels (doli- meso- and brachyfacial groups, skeletal Class I, II, and III). The GLM model was then set up so as to decompose the overall variance into fractions that are 
contributed to the mandible by the craniofacial factor (principal factor) as well as to possible differences of the principal factor across the different groups (interaction term). ${ }^{12,32}$

\section{Results}

Table 2 shows the results for the interaction strength. Except for the brachyfacial subsample, the PLS1 (singular warp) vector results indicated that the mandible was highly integrated with the cranium and explained between 57.8\% (Class II) and 68.1\% (total sexcorrected sample) of the total covariation. A higher correlation was found in Class III subjects $(\mathrm{r}=0.75, P<0.0001)$. For the brachyfacial subsample, the PLS1 vector was not significant and the PLS 2 vector, although significant, only explained 19\% of the craniummandible covariation.

Figure 2 shows the PLS1 covariation pattern between the mandible and cranium in the complete (sex-corrected) sample. Variations in the spatial configurations of the cranial base and maxilla (2D and 3D, because mid-line and bilateral landmarks were involved) were related to coordinated shape variations in the mandible. The spectrum of mandibular variations detected among human populations (e.g., open vs. closed corpus-ramus angle, narrow vs. wide ramus breadth, thick vs. thin symphysis profile, high vs. low coronoid process, convex vs. concave basal border, etc.) was associated with a cranial base swing, with Sella as the center, and a rectangular-to-quadrate maxilla variation. Thus, a long face occurred in the context of a vertically oriented cranial base and a deep and short maxilla. A long-face mandible showed an open corpus-ramus angle, narrow ramus breadth, and thin, high symphysis, whereas a short face displayed the opposite configuration.

These associations were further corroborated by the GLM analysis results (Table 3). In both analyses, correlations between the cranium-mandible PLS1 scores were highly 
significant. However, a highly significant interaction term was also identified in the GLM with vertical facial patterns as the group factor. This finding supported the hypothesis of different cranium-mandible covariation patterns in these groups. The interaction term was not significant between the skeletal classes, indicating similarity in the cranium-mandible covariation pattern (Fig. 3). Thus, all of the analyses suggested that the null hypothesis should be rejected with respect to facial patterns but not skeletal classes.

Because the integration patterns were different among the vertical facial patterns, we conducted separate PLS analyses for the dolicho- and brachyfacial subsamples (Fig. 4). As expected, the long-face subsample mostly followed the covariation scheme detected for the whole sample, but with a few differences. Notably, although the anterior part of the maxillary alveolar process remained stable, the posterior part (U2M) went down, with important occlusal repercussions. This cranium-face configuration was associated with a more retruded, hyperdivergent mandible and a narrower, elongated, and posteriorly orientated ramus.

A different cranium-mandible covariation pattern was found in the brachyfacial subsample. The anterior cranial base (i.e., presphenoid plane, cribriform plate, and Nasion), Frankfort plane position, and maxillary orientation remained almost stable. Variation was mostly concentrated in the posterior face and posterior base. This finding was related to an anteroposterior rotation of the basion, which led to cranial base flexion. U2M became more backwardly located as the cranial base was flexed, and the maxillary alveolar process became longer due to posterior elongation. The mandible responded with corpus-ramus angle and ramus breadth variations, presumably due to condylar growth. The anterior mandibular corpus and chin rotated anteriorly, leading to upward displacement of the chin, 
which moved closer to the maxilla. This movement contributed to shortening of the anterior facial height and anterior supraocclusion.

\section{Discussion}

Geometric morphometric methods and GLM were used to reveal the pattern and strength of MI between the cranium-face and mandible in adult subjects with skeletal Class I, II and III malocclusions, on the one hand, and meso- dolicho-, and brachyfacial configurations, on the other hand. The null hypothesis of no difference between groups was rejected for facial patterns but not for skeletal classes. The craniofacial-mandibular covariation did not differ between different skeletal classes, but the MI was different between dolicho- and brachyfacial subjects.

The level of covariation between the cranium and mandible were similarly high in the three skeletal classes, which explained the similarly large covariance between the two structures (57.80\% in Class II to 60\% in Class III). That could be interpreted as a prevalent influence $(\sim 60 \%)$ of the craniofacial configuration (e.g. interdependencies between anatomical systems) in the generation of sagittal (antero-posterior) skeletal malocclusion in the three skeletal classes. The remaining morphological component of the skeletal malocclusion ( 40\%) would be independent of this particular integration (PLS1) between the cranium and mandible. Because we are unable to act clinically over the cranial base, our ability to correct orthopedically sagittal skeletal malocclusions acting only in the mandible, is limited to, at most, this $40 \%$. From this perspective, the treatment of Class III malocclusions due to mandibular prognathism would be slightly more limited as the degree of cranium-mandible MI increases $(r=0.75)$, when compared with Class II malocclusions with a retrognatic-hypoplastic mandible $(r=0.71)$. Nevertheless, these analyses only 
account for morphological traits; in the genesis and correction of skeletal malocclusions involving the mandible other factors, such as neuromuscular balance and function, should also be considered. ${ }^{33}$

The cranium-mandible MI was significantly different between dolicho- and brachyfacial subjects, in terms of the pattern and strength of covariation. In dolichofacial subjects, the cranium and mandible were highly integrated, explaining $56.10 \%$ of the total covariation. In brachyfacial subjects, the degree of integration explained only $~ 19 \%$ of the total covariation. As a result, our ability to modify the mandible morphology by orthopedic approaches may be less limited in brachy- than in dolichofacial subjects because the interdependence between the cranium and mandible is much lower.

Dolicho- and brachyfacial subjects exhibited important differences in the degree of cranial base variations. In the most-dolichofacial configuration many traits varied: the sphenoid plane and cribriform plate were more upward, posterior cranial base was anteriorly rotated, Frankfurt plane was more downward, maxilla was decreased, retruded, and anterorotated, and posterior alveolar process was markedly decreased. Thus, the cranium became anteroposteriorly compressed, with a large variation at the M2 level. This craniofacial configuration was associated with a characteristic mandible: retruded and hyperdivergent, with a narrow, elongated, and posteriorly orientated ramus, pronounced preangular notch, decreased corpus height, and narrow, elongated, and vertically projecting symphysis.

In contrast, the range of cranial base variations was reduced in brachyfacial subjects. In the most-brachyfacial configuration, the anterior cranial base and Frankfurt plane remained almost invariable. Only the posterior cranial base varied, presenting an anteroposterior rotation. For the maxilla, only a slight vertical compression was detected. 
However, in the anteroposterior dimension, the maxilla was protruded and the alveolar process was elongated backward. This craniofacial configuration was also associated to a characteristic mandible: square-shaped, protruded and hypodivergent, with a wide, anteriorly oriented ramus, increased corpus and symphysis thickness, and anterorotation of the anterior mandibular corpus and chin, leading to upward projection.

Unique covariation patterns between the maxilla and mandible were observed in the dolicho- and brachyfacial groups. In the most-dolichofacial morphology, a decreased, anterorotated, and retruded maxilla with a decreased and anterorotated posterior alveolar process covaried with a more hyperdivergent mandible, whit an increased preangular notch, and posterior orientated ramus. This decreased posterior maxilla height may potentially be involved in the morphological development of a dolichofacial mandible. ${ }^{12}$ In contrast, in the most-brachyfacial morphology, a slightly protruded and compressed maxilla and backward elongation of the alveolar process covaried with anterorotation of the anterior mandibular corpus and chin, upward chin displacement, and anterorotated and wider ramus. The vertical compression and mainly, the backward elongation of the alveolar process could potentially be implicated in the morphological development of a brachyfacial mandible.

The ontogenetic processes underlying the findings of this study could have relevant clinical repercussions. For example, development of a dolichofacial mandible might be prevented by reducing the posterior vertical development of and posterorotating the maxilla, while compressing and stimulating the backward elongation of the alveolar process. Development of a brachyfacial mandible might be prevented by stimulating the vertical development of and anterorotating the maxilla, while shortening and elongating the maxillary alveolar process. However, the efficacy of such procedures depends on myriad 
factors, including the problem severity, morphological configuration of the cranial base, potential autonomous genetic control of the mandibular growth, oral functions, etc.

The observed similarity between skeletal classes and differences between facial patterns may explain why most of the specific traits of morphological covariation between the cranium and mandible in the total sample were related to the vertical dimension, whereas the sagittal (anteroposterior) dimension was almost unaffected along the PLS1 vector.

To summarize, dolicho- and brachi-facial subjects showed specific and different cranium-face and associated mandible configurations. The cranium-face configuration may have an important influence ( $60 \%$ ) on the generation of sagittal (anteroposterior) skeletal malocclusions. The remaining morphological component of the skeletal malocclusion ( $40 \%$ ) would be independent of this particular integration (PLS1) between the craniumface and mandible. Future studies should analyze 3D data from growing and adult subjects to identify the processes that cause the final morphological and functional integration between the cranial base, ethmomaxillary complex, and mandible. 


\section{ACKNOWLEDGMENTS}

This research was founded by the Spanish Ministry of Economy and Competitiveness (Projects CGL2012-36682 and CGL2012-37279). 


\section{REFERENCES}

1. Lieberman DE, Pearson OM, Mowbray KM. Basicranial influence on overall cranial shape. J Hum Evol 2000;38:291-315.

2. Klingenberg CP. Morphological integration and developmental modularity. Annu Rev Ecol Evol Syst 2008;39:115-132.

3. Mitteroecker P, Bookstein F. The evolutionary role of modularity and integration in the hominoid cranium. Evolution 2008;62:943-958.

4. Smith KK. Integration of craniofacial structures during development in Mammals. Am Zool 1996;36:70-79.

5. Cheverud JM. Phenotypic, Genetic, and Environmental Morphological Integration in the Cranium. Evolution 1982;36:499-516.

6. Marsico E, Gatto E, Burrascano M, Matarese G, Cordasco G. Effectiveness of orthodontic treatment with functional appliances on mandibular growth in the short term. Am J Orthod Dentofacial Orthop 2011;139:24-36.

7. Meikle MC. Remodeling the dentofacial skeleton: the biological basis of orthodontics and dentofacial orthopedics. J Dent Res 2007;86:12-24.

8. Shen G, Darendeliler MA. The adaptive remodeling of condylar cartilage---a transition from chondrogenesis to osteogenesis. J Dent Res 2005;84:691-699.

9. Alarcon JA, Bastir M, Rosas A, Molero J. Chincup treatment modifies the mandibular shape in children with prognathism. Am J Orthod Dentofacial Orthop 2011;140:38-43.

10. Saleh M, Hajeer MY, Al-Jundi A. Short-term soft- and hard-tissue changes following Class III treatment using a removable mandibular retractor: a randomized controlled trial. Orthod Craniofac Res 2012.

11. McCollum MA. The robust australopithecine face: a morphogenetic perspective. Science 1999;284:301-305.

12. Bastir M, Rosas A. Facial heights: evolutionary relevance of postnatal ontogeny for facial orientation and skull morphology in humans and chimpanzees. J Hum Evol 2004;47:359-381.

13. Ackermann RR. Ontogenetic integration of the hominoid face. J Hum Evol 2005;48:175-197.

14. McCane B, Kean MR. Integration of parts in the facial skeleton and cervical vertebrae. Am J Orthod Dentofacial Orthop 2011;139:e13-30.

15. Bastir M, Rosas A. Hierarchical nature of morphological integration and modularity in the human posterior face. Am J Phys Anthropol 2005;128:26-34.

16. Polanski JM. Morphological Integration of the Modern Human Mandible during Ontogeny. Int J Evol Biol 2011;2011:545879.

17. Enlow DH, Hans MG. Essentials of facial growth. . Philadelphia, London, New York: W.B. Saunders Company; 1996.

18. Enlow DH, Azuma M. Functional growth boundaries in the human and mammalian face. Birth Defects Orig Artic Ser 1975;11:217-230.

19. Bastir M, Rosas A, O'Higgins P. Craniofacial levels and the morphological maturation of the human skull. J Anat 2006;209:637-654.

20. Richtsmeier JT, Aldridge K, DeLeon VB, Panchal J, Kane AA, Marsh JL et al. Phenotypic integration of neurocranium and brain. J Exp Zool B Mol Dev Evol 2006;306:360-378.

21. Wellens HL, Kuijpers-Jagtman AM, Halazonetis DJ. Geometric morphometric analysis of craniofacial variation, ontogeny and modularity in a cross-sectional sample of modern humans. J Anat 2013;222:397-409.

22. McIntyre GT, Mossey PA. Size and shape measurement in contemporary cephalometrics. Eur J Orthod 2003;25:231-242. 
23. Halazonetis DJ. Morphometrics for cephalometric diagnosis. Am J Orthod Dentofacial Orthop 2004;125:571-581.

24. Rohlf FJ, Corti M. Use of two-block partial least-squares to study covariation in shape. Syst Biol 2000;49:740-753.

25. Bookstein FL, Gunz P, Mitteroecker P, Prossinger H, Schaefer K, Seidler H. Cranial integration in Homo: singular warps analysis of the midsagittal plane in ontogeny and evolution. J Hum Evol 2003;44:167-187.

26. Zelditch ML, Lundrigan BL, Garland T, Jr. Developmental regulation of skull morphology. I. Ontogenetic dynamics of variance. Evol Dev 2004;6:194-206.

27. Rohlf FJ, Corti M. The Use of Two-Block Partial Least-squares to Study Covariation in Shape. Systematic Zoology 2000;49:740-753.

28. Klingenberg CP. Morphometric integration and modularity in configurations of landmarks: tools for evaluating a priori hypotheses. Evolution and Development 2009;11:405-421.

29. Bastir M, Rosas A, Sheets DH. The morphological integration of the hominoid skull: A Partial Least Squares and PC analysis with morphogenetic implications for European Mid-Pleistocene mandibles. In: Slice D, editor. Modern Morphometrics in Physical Anthropology. New York: Kluwer Academic/Plenum Publishers; 2005. p. 265-284.

30. Steiner CC. Cephalometrics for you and me. Am J Orthod 1953;39:729-755.

31. Riolo ML, Moyers RE, McNamara JA Jr, Hunter WS. An atlas of craniofacial growth: Cephalometric standards from The University School Growth Study, The University of Michigan. Monograph 2, Craniofacial Growth Series, Center for Human Growth and Development. Ann Arbor, Mich: The University of Michigan; 1974.

32. Dobson AJ. An Introduction to Generalized Linear Models. London New York Washington DC: Boca Raton; 1990.

33. Gkantidis N, Halazonetis DJ, Alexandropoulos E, Haralabakis NB. Treatment strategies for patients with hyperdivergent Class II Division 1 malocclusion: is vertical dimension affected? Am J Orthod Dentofacial Orthop 2011;140:346-355. 